\title{
Health Plan Enrollment and Disenrollment of Individuals With and Without Established Chronic Disease in a U.S. Commercially Insured and Medicare Advantage Population
}

\author{
Haechung Chung, MPH; Gaurav Deshpande, PhD; Julia Zolotarjova, MSc; Ralph A. Quimbo, MS; \\ David M. Kern, PhD; Philip T. Cochetti, BA; and Vincent J. Willey, PharmD
}

\begin{abstract}
BACKGROUND: Chronic disease is associated with increased health care resource utilization and costs. Effective development and implementation of health care management and clinical intervention programs require an understanding of health plan member enrollment and disenrollment behavior.

OBJECTIVE: To examine the health plan enrollment and disenrollment behavior of commercially insured and Medicare Advantage members with established chronic disease compared with matched members without the disease of interest, using data from a large national health insurer in the United States.

METHODS: This retrospective matched cohort study used administrative claims data from the HealthCore Integrated Research Database from January 1,2006 , to November 30,2015 , to identify adults with chronic disease (type 2 diabetes mellitus [T2DM], cardiovascular disease [CVD], chronic obstructive pulmonary disease [COPD], rheumatoid arthritis [RA], and breast cancer $[\mathrm{BC}])$. Members with no established chronic disease (controls) were directly matched to members with established chronic disease (cases) on demographic characteristics. The earliest date on which members met the criteria for a given disease was defined as the index date. Controls had the same index date as the matched cases. All members had $\geq 12$ months of continuous health plan enrollment before the index date. Outcomes included health plan member disenrollment and enrollment duration. Incidence rates per 1,000 member-years for member disenrollment were evaluated along with incidence rate ratios (relative risk) using a Poisson model. Time to disenrollment was analyzed by Cox proportional hazard models and Kaplan-Meier survival curves. Sensitivity analyses were conducted where death was included as a disenrollment event.
\end{abstract}

RESULTS: 70,907 health plan members with BC $(99.7 \%$ female, mean age 60.5 years); 28,883 members with COPD (52.3\% female, mean age 66.7 ); 835,358 members with CVD (50.5\% female, mean age 62.7 years); 210,936 members with T2DM (45.2\% female, mean age 53.6 years); and 31,954 members with RA ( $72.0 \%$ female, mean age 55.5 years) were matched to controls and met the study criteria. The incidence rates of health plan disenrollment ranged from 155 to 192 members per 1,000 members per year. Compared with controls, members with chronic disease were $30 \%-40 \%$ less likely to disenroll from a health plan $(P<0.001$ for all comparisons). Among those who disenrolled, enrollment duration ranged from 2.3 to 2.7 years among cases and 1.5 to 1.8 years among matched controls $(P \leq 0.001$ for all comparisons).

CONCLUSIONS: This real-world study demonstrated that members with chronic disease had a significantly lower rate of disenrollment and a longer duration of enrollment compared with matched controls and were continuously enrolled for almost a year longer than members without a diagnosed chronic disease. Understanding health plan enrollment and disenrollment behavior may provide a valuable context for determining the time frame for the effect of health care programs and initiatives.

J Manag Care Spec Pharm. 2019;25(5):612-20

Copyright $\odot 2019$, Academy of Managed Care Pharmacy. All rights reserved.

\section{What is already known about this subject}

It has been well documented that individuals with chronic disease experience a higher use of health care services and greater health-related expenses than healthy individuals, suggesting health insurance coverage to be imperative for this population.

Health plan disenrollment data are available from multiple retrospective observational studies examining Medicare and Medicaid populations, but only a few studies have focused on members with chronic disease.

Studies that have assessed health plan retention and disenrollment behavior among members with chronic disease primarily examined individuals with a cancer diagnosis.

\section{What this study adds}

This study is the first to provide information on real-world health plan enrollment and disenrollment behavior among U.S. commercially insured and Medicare Advantage populations with a spectrum of established chronic disease.

Members with chronic disease were 30\%-40\% less likely to disenroll from a health plan, and for those who disenrolled, members with chronic disease were continuously enrolled in a health plan for approximately 2.5 years, almost a year longer than members without a diagnosed chronic disease.

The data generated from this study provide a valuable context for determining the time frame for the potential effect of health care management and clinical intervention programs for individuals with chronic disease in the United States.

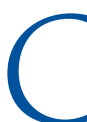
hronic diseases are among the most prevalent and costly health conditions, ${ }^{1,2}$ constituting a major cause of mortality and disability worldwide. ${ }^{3}$ In 2012, nearly half of adults (117 million) in the United States had at least 1 chronic health condition, and a quarter of adults experienced 
2 or more chronic health conditions. ${ }^{4}$ Leading contributors to the chronic disease epidemic include cardiovascular disease, cancer, chronic respiratory diseases, musculoskeletal diseases, and diabetes mellitus. ${ }^{5}$

There is a significant economic burden on the national health care system owing to an aging population that continues to grow and an increased risk for individuals to develop chronic disease at a younger age..$^{5-7}$ More than $\$ 2.3$ trillion $(86 \%)$ of health care expenditure was attributed to chronic disease in 2010. ${ }^{8,9}$ Projections indicate that chronic disease will remain a public health challenge, with total costs estimated to be $\$ 42$ trillion from 2016 to $2030{ }^{2}$

National data and findings from several studies suggest that individuals with chronic disease use more health care services and incur greater health-related expenses than healthy individuals. ${ }^{6,8,10-16}$ Health care spending for individuals with 1 chronic health condition has been documented to be almost 2.5 times higher than for those without a chronic health condition. ${ }^{8}$ The presence of multiple comorbid conditions has been shown to raise health care resource utilization and costs., ${ }^{8}, 10,16-19$ Individuals with chronic disease are vulnerable to high health care costs, and health coverage is imperative.

Health insurance coverage rates provided by the U.S. Census Bureau report that $67.5 \%$ (216.2 million) of the general U.S. population were commercially (privately) insured in 2016 with insurance provided mainly through an employer $(55.7 \%, 178.5$ million). ${ }^{20}$ Even though chronic disease is highly prevalent among the senior population, many individuals with chronic disease are of working age and privately insured. ${ }^{6}$ Notably, the number of older personnel in the workforce has risen and may potentially be attributed to the need for acquisition of private health insurance coverage. ${ }^{21,22}$ Since the introduction of the Affordable Care Act (ACA) in 2010, health insurance coverage has expanded allowing more individuals with chronic disease to have access to health services. ${ }^{23,24}$ Individuals with chronic disease are likely to seek a health plan that provides continued medical coverage, choice of health care providers, ${ }^{25}$ and financial security due to greater health needs. ${ }^{8,10,23}$

It can be suggested that personal factors and the presence of a chronic disease may influence individuals' decisions to enroll or disenroll from a health plan. Reasons that may prompt disenrollment include loss of employment (average employee tenure is 4.2 years $^{26}$ ), age, and increased medical support required to manage comorbid conditions. ${ }^{8,10,22,27}$ Individuals who also have poor health or remain insured for a longer duration may be less likely to disenroll from a health plan. ${ }^{27}$

Although health care resource utilization and the cost burden associated with chronic disease are well documented, there were no studies found that have investigated the duration of health plan enrollment and the rate of disenrollment among individuals with chronic disease in a commercially insured and Medicare Advantage population. An understanding of health plan member enrollment and disenrollment behavior would provide a better insight into the development and longterm benefits of chronic disease care management initiatives and could influence health policy decisions. In addition, this information would be of interest and value to researchers in their preparation and planning of studies using administrative claims data, since the utility of such data is directly tied to the duration of health plan enrollment as a means to observationally measure study endpoints and outcomes.

The current study was designed to determine and quantify the relative influence of 5 common chronic diseases (type 2 diabetes mellitus [T2DM], cardiovascular disease [CVD], chronic obstructive pulmonary disease [COPD], rheumatoid arthritis $[\mathrm{RA}]$, and breast cancer $[\mathrm{BC}]$ ) on the health plan enrollment and disenrollment behavior of a commercially insured and Medicare Advantage population.

\section{Methods}

\section{Study Design and Population}

This retrospective matched cohort study identified members from the HealthCore Integrated Research Database (HIRD). HIRD includes administrative claims for 14 commercial and Medicare Advantage insurance plans and, at the time of this study, contained longitudinal claims data for more than 38 million enrollees from all U.S. census regions. Continuous health plan enrollment (eligibility segment) information is available in the HIRD, including the start and end date of members' continuous eligibility.

Members enrolled in a commercial or Medicare Advantage health plan with the disease areas of interest (T2DM, CVD, COPD, RA, and BC) were matched to controls without the given disease. Matched controls may have more than 1 disease and belong to other chronic disease cohorts. Members with the disease of interest and their respective controls were identified during the study period from January 1, 2006, to December 31,2015 , in the HIRD. The management of all data and study materials conformed with the Health Insurance Portability and Accountability Act (HIPAA) rules. A limited dataset, which excluded patient-identifying information, was used for all analyses, as defined by the HIPAA Privacy Rule.

\section{Patient Selection}

Members with the given disease of interest (cases) and the respective control pool with medical and pharmacy eligibility were identified separately for each chronic disease. The index date was assigned as the earliest date on which members met all the criteria for the given disease condition during the study intake period between January 1, 2007, and December 31, 2014, and, by design, represents the earliest point of disease onset to demarcate the start of the follow-up among study cases. In the absence of a cohort-defining event to demarcate the study follow-up, patients in the control pool were assigned an index 
date equal to that of their matched cases as part of the matching process. All members were required to have $\geq 12$ months of continuous health plan enrollment before the index date.

Patients with CVD aged 18 years or older were identified as individuals with $\geq 1$ inpatient or emergency department claims with CVD (primary or secondary) or CVD-related procedure or $\geq 2$ claims for CVD outpatient claims with CVD events or $\geq 1$ pharmacy claim for a P2Y12 inhibitor. CVD events were defined as myocardial infarction (International Classification of Diseases, Ninth Revision, Clinical Modification [ICD-9-CM] diagnosis codes: 410.xx, 412.xx); stable and unstable angina (ICD-9-CM diagnosis codes: 413.xx, 411.1x, 411.81); coronary atherosclerosis (ICD-9-CM diagnosis code: 414.xx); coronary revascularization (ICD-9-CM procedure codes: 00.66, 36.0x-36.3x; Common Procedural Terminology, 4th Edition [CPT-4]/Healthcare Common Procedure Coding System [HCPCS]: 33510-33523, 33533-33536, 92980-92982, 92984, 92995, 92996, S2205-S2209, G0290, G0291); ischemic stroke (ICD-9-CM diagnosis codes: 433.xl, 434.xx, 436.xx, $437.0 x, 437.1 x, 438.0 x-438.12,438.19-438.89$; ICD-9-CM procedure codes: 38.12; CPT-4/HCPCS: 35301, 35390); peripheral artery disease (ICD-9-CM diagnosis codes: 440.xx, 433.x0, 441.3x, 441.4x, 443.9x, 445.0x; ICD-9-CM procedure codes: $00.63,00.55,00.61,00.64,39.50,39.72,39.74,39.90,38.13$, 38.18; CPT-4/HCPCS: 34800-34805, 37215-37216, 93668, $35450-35459, \quad 35470-35475, \quad 35480-35485,35490-35495$, $35501-35571, \quad 35583-35587,35601-35671,37205-37208$, 35081-35103, 37220-37235, 35301); and use of P2Y12 inhibitors.

Patients with T2DM aged 18 years or older were identified as individuals with $\geq 1$ claim with a T2DM diagnosis (ICD9-CM diagnosis codes: 250.x0, 250.x2) and $\geq 1$ fill for oral antidiabetic medication. Patients with a diagnosis of type 1 diabetes or gestational diabetes were excluded.

COPD patients aged 40 years or older were identified as individuals with $\geq 1$ inpatient visit with a primary diagnosis for COPD (ICD-9-CM diagnosis code: 491.xx, 492.xx, 496. $\mathrm{xx}$ ), $\geq 1$ emergency room visit with a COPD diagnosis (irrespective of primary diagnosis), or $\geq 2$ medical claims with a COPD diagnosis (irrespective of primary diagnosis). RA patients aged 18 years or older were identified as individuals with $\geq 2$ claims with a diagnosis for RA (ICD-9-CM diagnosis code: 714.0x) and $\geq 1$ claim for disease-modifying antirheumatic drugs. BC patients aged 18 years or older were identified as individuals with $\geq 3$ claims with a breast cancer diagnosis (ICD-9-CM diagnosis code: 174.xx).

The control pool for each disease of interest included members with $\geq 12$ months of continuous health plan enrollment and those who did not meet any patient selection criteria for the given disease. Members with the disease of interest were directly matched 1:1 to a control based on age; gender; health plan type (health maintenance organization, preferred provider organization, and consumer-driven health plan); ACA plan enrollment; residence region; primary policy holder status; and length of pre-index continuous health plan enrollment in 6-month increments up to 60 months or more.

The matched control was required to have continuous health plan enrollment overlapping the index date for the case. The index date for the control was set as the same index date as that of the matched case. The matched control was required to meet the age criterion for the given disease along with $\geq 12$ months of continuous health plan enrollment before the index date. The matched control could not have the given disease before the index date of the case but could develop the condition following the paired index date.

\section{Outcome Measures}

Study outcomes that included disenrollment and enrollment duration were reported among cases and their matched controls.

Disenrollment was defined as the end date of the continuous eligibility segment occurring before or at the end of the study period, not including death. Two separate sensitivity analyses were conducted where death was included as a disenrollment event in addition to the criteria stated above and by excluding members who disenrolled or died during the first 30 days of follow-up.

For the main and sensitivity analyses, enrollment duration was defined as the time elapsed between the index date and disenrollment.

\section{Statistical Analysis}

Descriptive statistics were reported for continuous and categorical data. The frequency and proportion of members with disenrollment during the post-index follow-up period were reported descriptively for the case and matched control cohorts. Observation ended at the earliest of either the date of disenrollment or loss to follow-up (due to death or end of the study period). For the members in the control group, censoring also occurred at the earliest point of meeting the criteria for the given disease resulting in end of follow-up.

The incidence rate of member disenrollment was calculated as the number of members with disenrollment at any time during the post-index period divided by the number of total person-years of post-index observation. The unadjusted incidence rates were reported as an incidence rate of member disenrollment per 1,000 member-years. The relative risk of disenrollment was calculated as the rate ratio of the incidence rate of disenrollment for the case cohort over the incidence rate for the matched control cohort.

Incidence rate ratio (RR, or relative risk) with a $95 \%$ confidence interval (CI) was calculated via a Poisson model with a robust error variance, log link, offset by log of the length of post-index follow-up time. The mean difference in enrollment duration between the case and matched control cohorts was analyzed using a negative binomial model with a log-link 
function. Time to disenrollment was analyzed using a Cox proportional hazards model along with a Kaplan-Meier survival curve. Statistical testing was performed for the matched pairs, and a $P$ value of $<0.05$ was considered as statistically significant. All statistical analyses were conducted using SAS Enterprise Guide 7.1 (SAS Institute, Cary, NC).

\section{Results}

\section{Patient Population}

We identified 33.9 million members with $\geq 1$ day health plan enrollment from the HIRD between January 1, 2007, and December 31, 2014. Among those patients, we identified 71,731 members with BC; 334,082 members with COPD; 850,772 members with CVD; 214,852 members with T2DM; 32,365 members with RA who qualified for matching from the HIRD during the study period.

A total of 70,907 health plan members with BC; 328,883 members with COPD; 835,358 members with CVD; 210,936 members with T2DM; and 31,954 members with RA were matched to controls (Appendix A, available in online article).

\section{Patient Characteristics}

Full patient characteristics are displayed in Appendix B (available in online article). Key patient characteristics are summarized below by chronic disease state.

Breast Cancer. Mean age of the BC matched cohort was 60.5 years; $99.7 \%$ were female; $0.4 \%$ held ACA exchange plans; and $76.5 \%$ were primary insurance policy holders. The proportion of patients enrolled in a Medicare Advantage plan was similar between the matched groups (non-BC 12.0\% vs. BC 12.6\%). The Deyo-Charlson Comorbid Index (DCI) score was similar among $\mathrm{BC}$ and non-BC members (0.8 vs. 0.7).

Chronic Obstructive Pulmonary Disease. Among the COPD matched cohort, the mean age of members was 66.7 years; $52.3 \%$ were female; $0.2 \%$ held ACA exchange plans; and $86.0 \%$ were primary insurance policyholders. The proportion of patients enrolled in a Medicare Advantage plan was slightly higher among COPD members compared with non-COPD members (20.6\% vs. 19.2\%). Also, COPD members had more comorbid conditions relative to non-COPD members (DCI: 1.6 vs. 0.9).

Cardiovascular Disease. Mean age of the CVD matched cohort was 62.7 years; $50.5 \%$ were female; $0.3 \%$ held ACA exchange plans; and $83.6 \%$ were primary insurance policyholders. The proportion of patients enrolled in a Medicare Advantage plan was similar between the matched groups (non-CVD 16.2\% vs. CVD 15.7\%). CVD members had more comorbid conditions compared with non-CVD members (DCI: 1.0 vs. 0.6).

Type 2 Diabetes Mellitus. In the T2DM matched cohort, the mean age of members was 53.6 years; $45.2 \%$ were female; $0.4 \%$

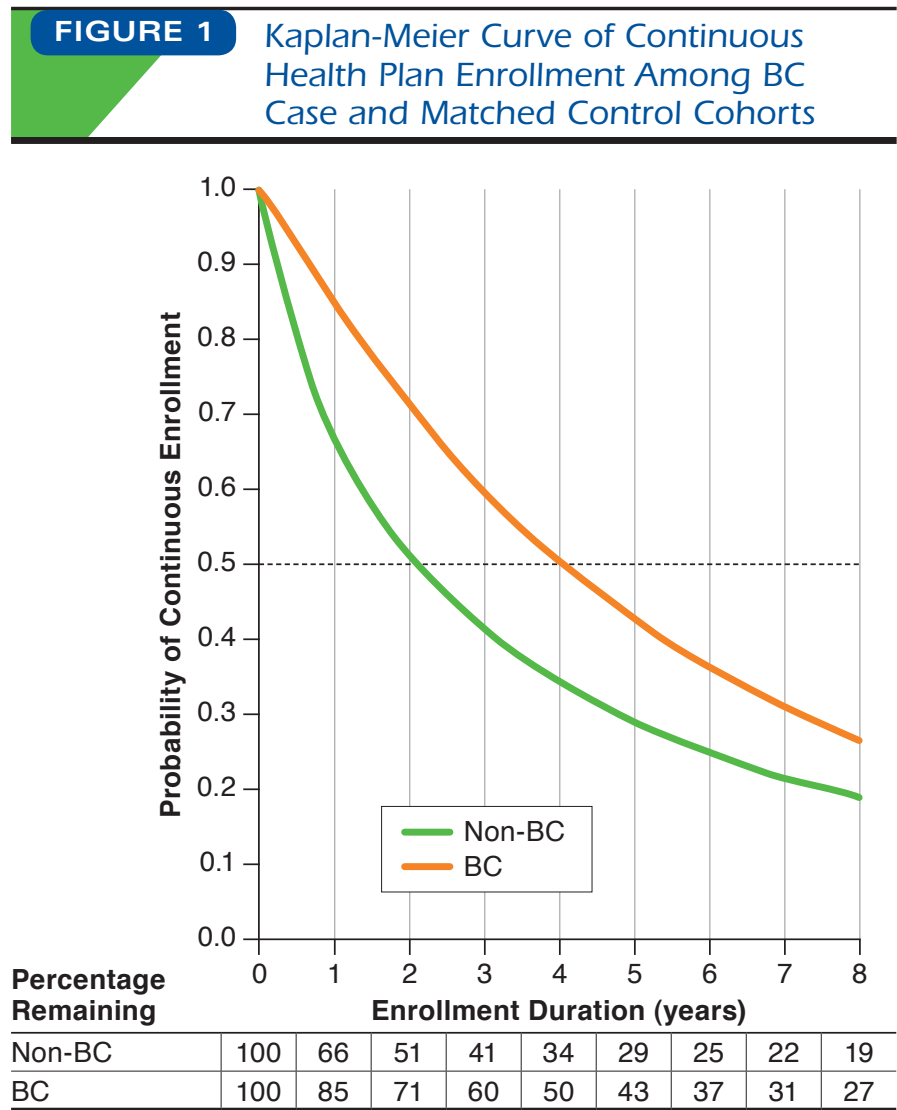

$B C=$ breast cancer.

held ACA exchange plans; and $78.3 \%$ were primary insurance policyholders. The proportion of patients enrolled in a Medicare Advantage plan was slightly higher among T2DM members compared with non-T2DM members (7.3\% vs. 6.4\%). The DCI score was similar between T2DM and non-T2DM members (0.8 vs. 0.7$)$.

Rheumatoid Arthritis. Among the RA matched cohort, the mean age of members was 55.5 years; $72.0 \%$ were female; $0.3 \%$ held ACA exchange plans; and $74.2 \%$ were primary insurance policyholders. The proportion of patients enrolled in a Medicare Advantage plan was similar between the matched groups (non-RA 10\% vs. RA 11\%). RA members had more comorbid conditions than non-RA members (DCI: 0.9 vs. 0.6).

\section{Member Disenrollment and Enrollment Duration}

Breast Cancer. The main analysis found that members with BC were $40 \%$ less likely to disenroll during follow-up compared with non-BC members (rate per 1,000 member year: 167 vs. $284 ; \mathrm{RR}=0.60,95 \% \mathrm{CI}=0.58-0.60 ; P<0.001$ ). Among those who disenrolled, time to disenrollment was 1.6 times as long among members with BC (mean, 2.4 vs. 1.5 years). Using a survival analysis approach (Figure 1), Cox regression showed 

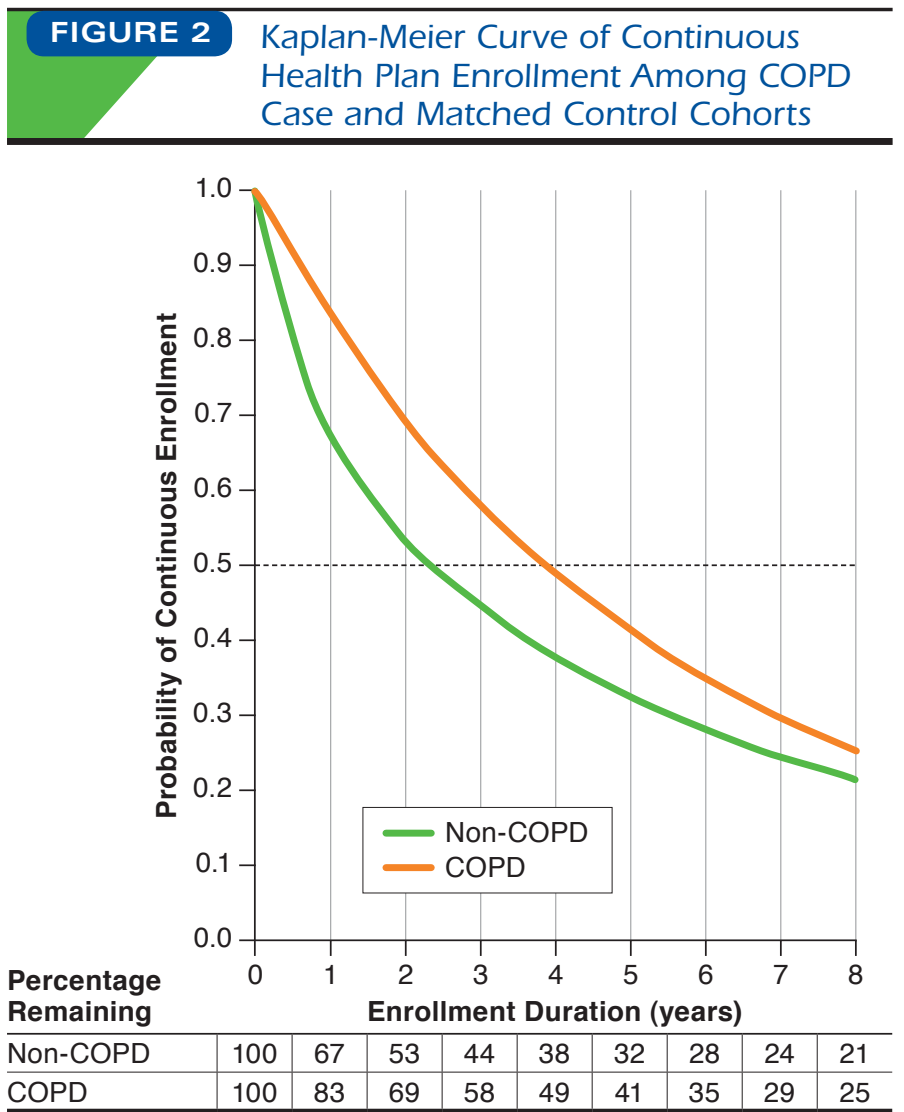

$\mathrm{COPD}=$ chronic obstructive pulmonary disease.

similar results (hazard ratio $[\mathrm{HR}]=0.61,95 \% \mathrm{CI}=0.61-0.62$; $P<0.001)$.

Disenrollment results from the primary analysis were consistent across all sensitivity analyses: (a) including death as a disenrollment event and (b) excluding patients who disenrolled or died during the first 30 days of follow-up at $P<0.001$.

Chronic Obstructive Pulmonary Disease. The main analysis found that members with COPD were 30\% less likely to disenroll during follow-up compared with non-COPD members (rate per 1,000 member year: 178 vs. 258 ; $R R=0.70,95 \%$ $\mathrm{CI}=0.69-0.70 ; P<0.001)$. Among those who disenrolled, time to disenrollment was 1.6 times as long among COPD members (mean, 2.4 vs. 1.5 years). Using a survival analysis approach (Figure 2), Cox regression showed similar results (HR=0.71, 95\% CI $=0.70-0.71 ; P<0.001)$.

Disenrollment results from the sensitivity analysis demonstrate a decrease in the relative risk and hazard of disenrollment between study cohorts at $P<0.001$.

Cardiovascular Disease. The main analysis found that members with CVD were 37\% less likely to disenroll during followup compared with non-CVD members (rate per 1,000 member
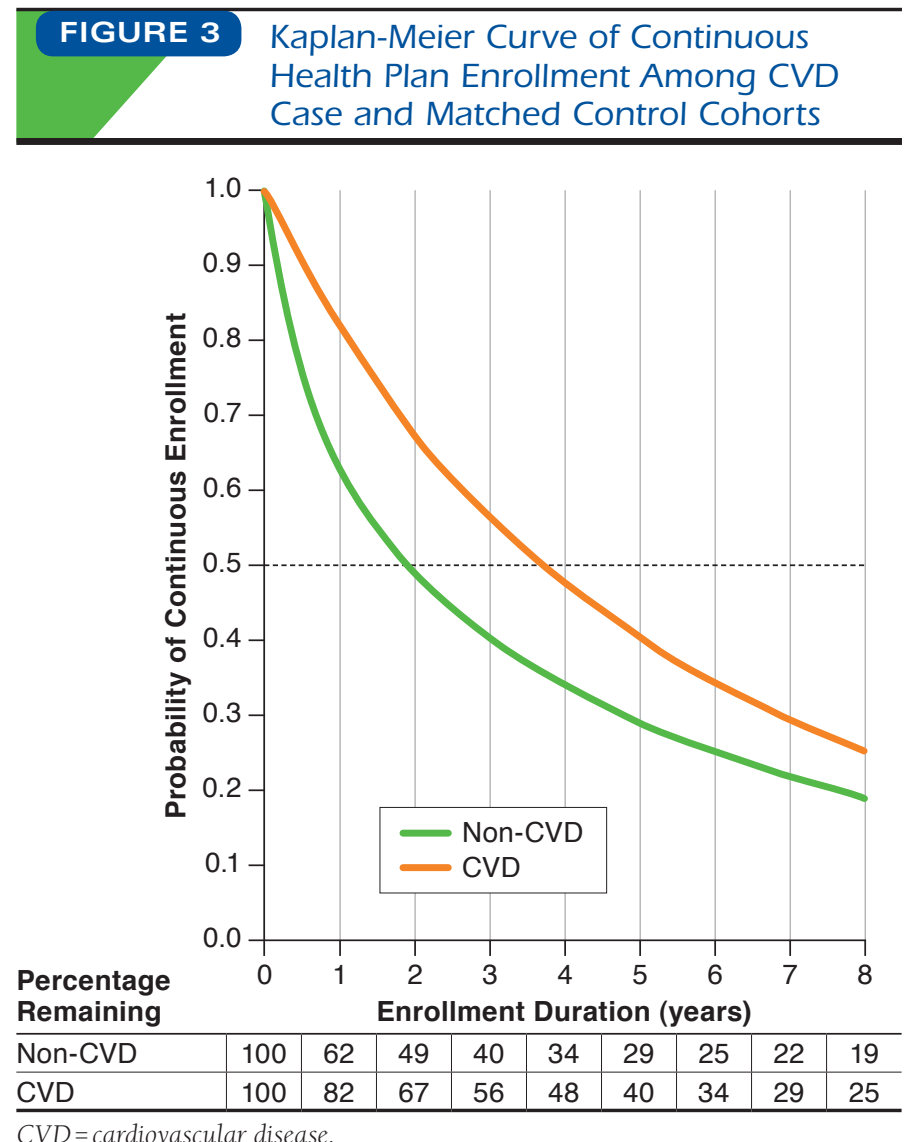

year: 183 vs. $292 ; \mathrm{RR}=0.63,95 \% \mathrm{CI}=0.62-0.63 ; P<0.001$ ). Among those who disenrolled, time to disenrollment was 1.7 times longer for CVD members (mean 2.3 vs. 1.5 years). Using a survival analysis approach (Figure 3), Cox regression showed similar results $(\mathrm{HR}=0.65,95 \% \mathrm{CI}=0.65-0.66 ; \mathrm{P}<0.001)$.

Disenrollment results from the sensitivity analysis showed a decrease of disenrollment between study cohorts at $P<0.001$.

Type 2 Diabetes Mellitus. The main analysis found that members with T2DM were $41 \%$ less likely to disenroll during follow-up compared with non-T2DM members (rate per 1,000 member year: 192 vs. 329; $\mathrm{RR}=0.59,95 \% \mathrm{CI}=0.58-0.59$; $P<0.001)$. Among those who disenrolled, time to disenrollment was 1.6 times as long among cases (mean, 2.4 vs. 1.5 years). Using a survival analysis approach (Figure 4), Cox regression showed similar results $(\mathrm{HR}=0.61,95 \% \mathrm{CI}=0.60$ $0.61 ; P<0.001$ ).

Disenrollment results from the primary analysis were consistent across all sensitivity analysis at $P<0.001$.

Rheumatoid Arthritis. The main analysis found that members with RA were $40 \%$ less likely to disenroll during follow-up compared with non-RA members (rate per 1,000 member year: 

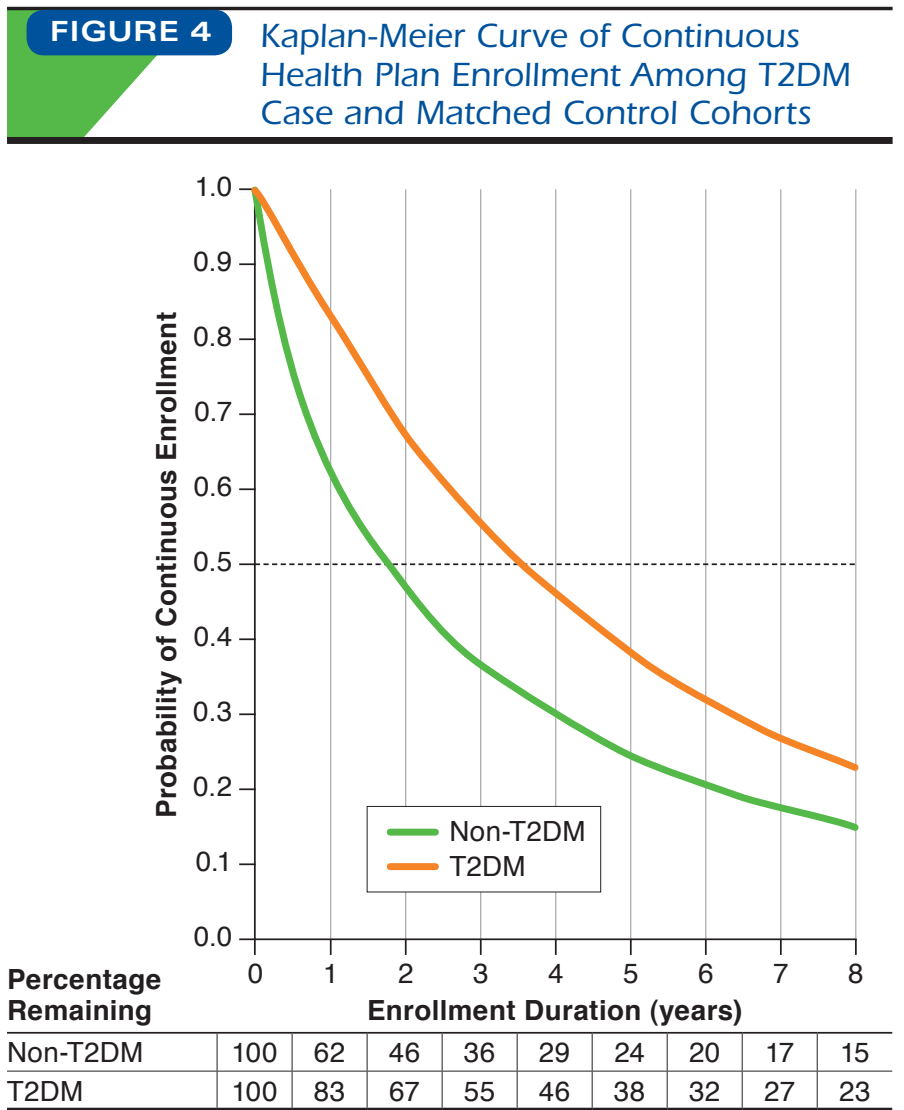

T2DM = type 2 diabetes mellitus

155 vs. $259 ; \mathrm{RR}=0.60,95 \% \mathrm{CI}=0.59-0.61 ; P<0.001)$. Among those who disenrolled, time to disenrollment was 1.5 times as long among RA members (mean, 2.7 vs. 1.8 years). Using a survival analysis approach (Figure 5), Cox regression showed similar results ( $\mathrm{HR}=0.61,95 \% \mathrm{CI}=0.60-0.62 ; \mathrm{P}<0.001)$.

Disenrollment results from the primary analysis were consistent across both sensitivity analyses at $P<0.001$.

\section{Discussion}

Continuity of enrollment within a health plan can influence effectiveness and return on investment associated with health care management and clinical intervention activities. Many real-world evidence studies use administrative claims data for which patients' continuity of coverage serves as an important parameter for study inclusion. Considering that many health care programs and research studies focus on chronic disease, an understanding of health plan enrollment and disenrollment behavior of members with chronic disease in comparison to the overall health plan population is essential. This information is also important to guide other health plan activities, such as year-to-year attribution of members in value-based payment
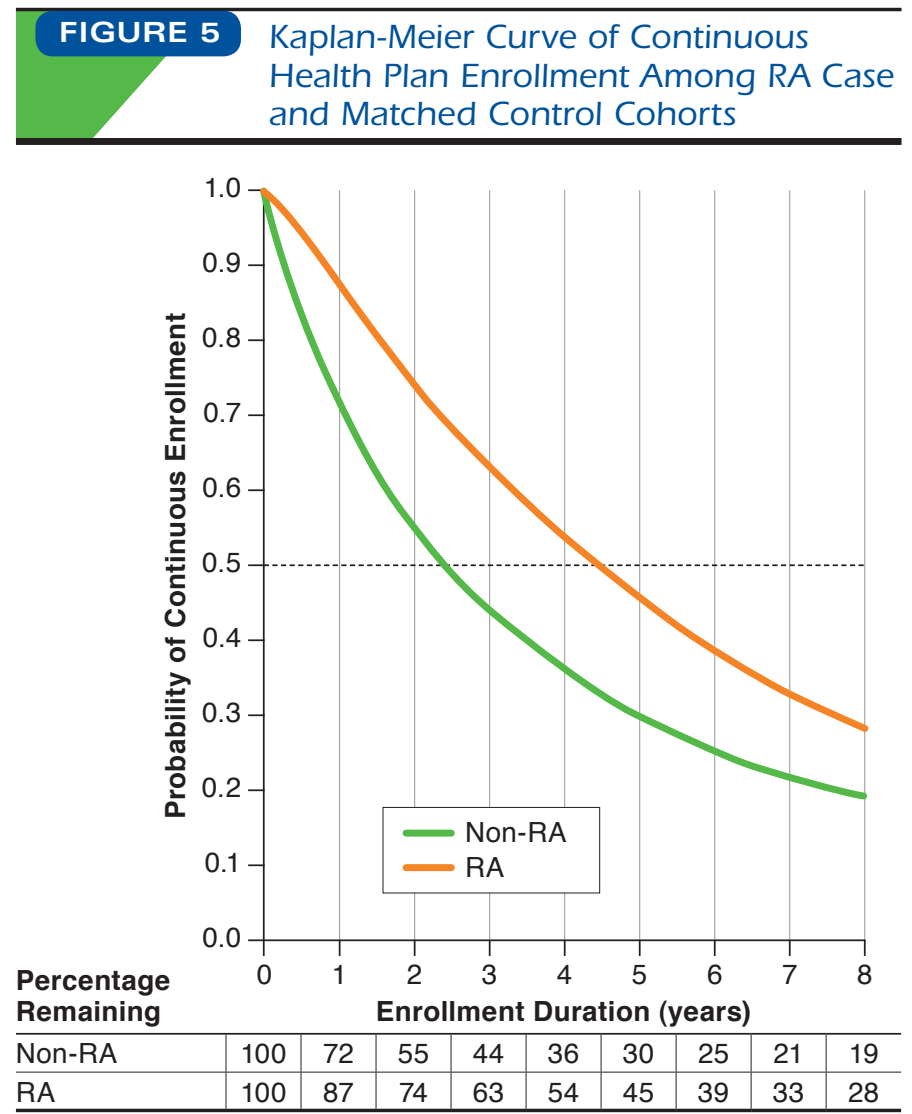

RA= rheumatoid arthritis

models and structuring multiyear initiatives such as chronic care management and member incentives.

To provide insight into health plan member behavior, this study examined the health plan enrollment and disenrollment behavior of members with various chronic diseases in a commercially insured and Medicare Advantage population using data from a large U.S. national health insurer. Members with BC, COPD, CVD, T2DM, and RA were 30\%-40\% less likely to disenroll from a health plan over the 10-year study period compared with members with similar demographic characteristics but without the presence of the diagnosed chronic condition corresponding to the matched case. Among those who disenrolled, members with a chronic condition remained continuously enrolled in a health plan for approximately 2.5 years, almost a year longer than members without a chronic condition. Given the long-term nature of these diseases, this additional period of enrollment can allow more time for return on investment in health care management initiatives.

When reviewing the results of the survival analysis, which takes into account the full population instead of those who disenrolled, there was approximately a 2 -year 
difference when one half of the members had disenrolled between members with and without the conditions of interest. In addition, results from the sensitivity analyses (which included mortality as a disenrollment event) were comparable; members with a chronic condition were significantly less likely to disenroll from a health plan. Prominently, the magnitude of the relative risk of disenrollment between cases and controls decreased when evaluating members with COPD and CVD, potentially attributed to an elevated mortality risk associated with these conditions.

To our knowledge, there are no studies that have explored the effect of a spectrum of chronic conditions on health plan disenrollment in a commercially insured population. Multiple studies have examined health plan disenrollment patterns among various Medicare and Medicaid populations, ${ }^{28-32}$ but only some studies had a concerted focus on chronic conditions. ${ }^{32-35}$

Our findings closely corroborate health plan disenrollment trends reported by Elkin et al. (2008) and Field et al. (2008). ${ }^{33,34}$ Based on available literature, the study by Elkin et al. applied the most relevant study design in reference to our study and examined health plan disenrollment among Medicare managed care enrollees aged 65 years or older with and without a cancer diagnosis (spectrum of diagnoses observed: breast, colorectal, prostate, or lung cancer), based on data from cancer registry records. ${ }^{33}$ Individuals with cancer were matched to cancer-free enrollees on the basis of baseline criteria, such as age, gender, race, and geographic location. The results of this study indicated that individuals with cancer were less likely to disenroll from Medicare managed care than matched cancerfree enrollees. ${ }^{33}$

Notably, our study includes several disease states that represent a diverse cross-section in an attempt to best represent the fuller spectrum of chronic conditions. The selected conditions varied by multiple attributes, such as the age of the members; treatments used (ranging from traditional medications to chemotherapy and biologics to medical and surgical procedures); prevalence; treatment by physician types; levels of acuity; and differing symptomatology. Our goal was to select a finite number of chronic conditions from which the results could be generalized across a wide array of diseases based on these attributes.

Although there were no formal comparisons between chronic conditions, several observations are worthy to note. Although chronic conditions did purposefully vary in the selection attributes, the incidence rates of health plan disenrollment (ranging from 155 to 192 members per 1,000 members per year) and enrollment duration (ranging from 2.3 to 2.7 years) were qualitatively similar. For these endpoints, RA was associated with the longest duration of enrollment. Although speculative in nature, this may be due to the concern over how a switch in health plan may affect the reimbursement for high-cost medications, including biologics, associated with the treatment of RA.

The relative risk of disenrollment was very similar between BC, CVD, T2DM, and RA members (ranging from 0.59 to 0.63), indicating that members with these conditions were between $37 \%$ and $41 \%$ less likely to disenroll relative to their controls. Notably, members with COPD were only 30\% less likely to disenroll relative to their controls $(\mathrm{RR}=0.70)$, which is a departure from the other chronic conditions. This finding may be attributed to the fact that COPD members and their matched controls were older than members in the other chronic condition cohorts, which also increased the proportion of members possessing Medicare coverage.

Importantly, individuals may disenroll from a health plan for a variety of reasons. The selection of a health plan for many commercially insured individuals is performed by an employer. If an employer switches health insurers, employees will become disenrolled from their current plan unless they leave their jobs or independently pursue the purchase of health insurance. In contrast, individuals may choose to stay at their current jobs to retain benefits from the insurer that their employer has elected. Occasionally, individuals may value this work benefit equally or above others and remain in their position due to the fear of losing health plan coverage. This phenomenon has been described as "job lock." Health status has been documented to be a significant determinant of being in a state of job lock. ${ }^{36-38}$ Employees who have high medical expenses due to a chronic condition are exceedingly susceptible to insurance-related job lock. ${ }^{37}$ Among employees with employer-provided health insurance, the presence of a chronic disease has been shown to reduce job mobility by up to $40 \%$ compared with employees who did not have employer-based coverage. ${ }^{37}$ Job lock may have undesirable personal and societal implications by limiting individual life choices and quality of life, as well as hindering an efficient labor market. ${ }^{37,38}$

In this study, the aforementioned differences in health plan disenrollment were statistically significant, as well as meaningfully different from a health care management, policy, and research perspective. Furthermore, a quantified understanding of the length of time that members with chronic conditions remain insured with a health plan can play a vital role in determining what types of health care management programs should be implemented. These programs, especially in the context of chronic conditions, may take an extended period of time (years) to have an influence on clinical, economic, and societal outcomes. Health plans can use these data to develop interventions that can deliver results within a time frame consistent with health plan enrollment behavior for their customers, whether that be employers or individual members.

The outcomes of this study suggest that attention should be given to individuals with chronic conditions when evaluating health plan enrollment and disenrollment behavior. 
Generalizing the enrollment duration for a particular group of members based on the overall population could significantly underestimate true enrollment time, which may lead to illinformed decisions with regards to health care management initiatives and clinical interventions.

\section{Limitations}

There are several limitations associated with administrative claims studies. First, coding errors of omission and commission and unreliable billing coding may have influenced the composition of the case and control cohorts. For instance, to be part of the $\mathrm{BC}$ cohort, members were required to have $\geq 3$ claims with ICD-9-CM diagnosis code 174.xx, which is a specific code for female breast cancer; yet, male patients were still included in the cohort-this is likely a result of coding error.

Second, our study included few members with coverage under an ACA exchange plan, and the results should not be generalized to those individuals. Future research in this area should include a larger sample of these individuals to assess the effect of the ACA on disenrollment behavior.

Third, an inherent outcome of our study included the increased comorbidity burden of the chronic conditions cohort compared with matched controls. A greater presence of comorbid conditions could add to the decreased likelihood of disenrollment among those with chronic conditions relative to controls. Future research in this area that can more effectively control for this difference would help in precisely estimating the variation in disenrollment risk that is attributable to the chronic disease of interest.

Finally, the outcomes of this study are based on data from commercially insured and Medicare Advantage members insured by 14 state plans in the United States. Therefore, the results may not be generalizable to individuals with other types of health insurance.

\section{Conclusions}

This real-world study was the first study to our knowledge to quantify the health plan enrollment and disenrollment behavior of commercially insured and Medicare Advantage members with various chronic conditions (BC, COPD, CVD, T2DM, and RA). Members with chronic conditions had a significantly lower rate of disenrollment and a longer duration of enrollment compared with matched controls. The health plan enrollment and disenrollment behavior data generated from this study should be used by health plan personnel responsible for the development and implementation of health care management and clinical intervention programs. These data provide a valuable context for determining the time frame for the potential effect of such programs. Importantly, a greater understanding of member behavior will allow health programs and initiatives to be better tailored. This has the potential to enhance the quality of health care decisions and policies, which can result in improved member health outcomes.

\section{Authors}

HAECHUNG CHUNG, MPH; GAURAV DESHPANDE, PhD JULIA ZOLOTARJOVA, MSc; RALPH A. QUIMBO, MS; and VINCENT J. WILLEY, PharmD, HealthCore, Wilmington, Delaware. DAVID M. KERN, PhD, Janssen Research and Development, Titusville, New Jersey, and PHILIP T. COCHETTI, BA, Department of Biostatistics, Epidemiology, and Informatics, University of Pennsylvania, Philadelphia.

AUTHOR CORRESPONDENCE: Haechung Chung, MPH, HealthCore, 123 Justison St., Ste. 200, Wilmington, DE 19801. Tel.: 302.230.2085; E-mail: jchung@healthcore.com.

\section{DISCLOSURES}

Funding for this study was provided by HealthCore, a wholly owned subsidiary of Anthem. Chung, Deshpande, Zolotarjova, Quimbo, and Willey are employees of HealthCore. Kern and Cochetti are former employees of HealthCore. Quimbo, Cochetti, and Willey are shareholders of Anthem. HealthCore receives funding from multiple pharmaceutical companies to perform various research studies outside of the submitted work.

The preliminary results of this study were presented at AMCP Nexus 2015; March 26-29, 2015; Orlando, FL, and the International Society for Pharmacoeconomics and Outcomes Research (ISPOR) 2017 Conference; May 20-24, 2017; Boston, MA

\section{REFERENCES}

1. Centers for Disease Control and Prevention. About chronic diseases. 2019. Available at: https://www.cdc.gov/chronicdisease/about/index.htm. Accessed April 10, 2019.

2. Partnership to Fight Chronic Disease. What is the impact of chronic disease on America? Available at: http://www.fightchronicdisease.org/sites/ default/files/pfcd_blocks/PFCD_US.FactSheet_FINAL1\%20\%282\%29.pdf. Accessed March 21, 2019

3. World Health Organization. Noncommunicable diseases. 2018. Available at: https://www.who.int/en/news-room/fact-sheets/detail/noncommunicablediseases. Accessed April 10, 2019.

4. Ward BW, Schiller JS, Goodman RA. Multiple chronic conditions among US adults: a 2012 update. Prev Chronic Dis. 2014;11:E62.

5. Prince MJ, Wu F, Guo Y, et al. The burden of disease in older people and implications for health policy and practice. Lancet. 2015;385(9967):549-62

6. Anderson G, Horvath J. The growing burden of chronic disease in America. Public Health Rep. 2004;119(3):263-70.

7. World Health Organization. Widespread misunderstandings about chronic disease-and the reality. 2005. Available at: http://www.who.int/chp/ chronic_disease_report/media/Factsheet2.pdf. Accessed March 21, 2019.

8. Gerteis J, Izrael D, Deitz D, et al. Multiple chronic conditions chartbook AHRQ Publications no. 14-003. 2010. Available at: https://www.ahrq.gov/ sites/default/files/wysiwyg/professionals/prevention-chronic-care/decision/ mcc/mccchartbook.pdf. Accessed March 21, 2019.

9. Iuga AO, McGuire MJ. Adherence and health care costs. Risk Manag Healthc Policy. 2014;7:35-44.

10. Buttorff C, Ruder T, Bauman M. Multiple chronic conditions in the United States. 2017. Available at: https://www.rand.org/content/dam/rand/ pubs/tools/TL200/TL221/RAND_TL221.pdf. Accessed March 21, 2019.

11. Bahler C, Huber CA, Brungger B, Reich O. Multimorbidity, health care utilization and costs in an elderly community-dwelling population: a claims data based observational study. BMC Health Serv Res. 2015;15(1):23. 
12. Paez KA, Zhao L, Hwang W. Rising out-of-pocket spending for chronic conditions: a ten-year trend. Health Aff. (Millwood). 2009;28(1):15-25.

13. Lehnert T, Heider D, Leicht H, Heinrich S, Corrieri S, Luppa M. Review: health care utilization and costs of elderly persons with multiple chronic conditions. Med Care Res Rev. 2011;68(4):387-420.

14. Basu J, Avila R, Ricciardi R. Hospital readmission rates in U.S. states: are readmissions higher where more patients with multiple chronic conditions cluster? Health Serv Res. 2016;51(3):1135-51.

15. Barnett ML, Hsu J, McWilliams JM. Patient characteristics and differences in hospital readmission rates. JAMA Inter Med. 2015;175(11):1803-12.

16. Mudge AM, Kasper K, Clair A, et al. Recurrent readmissions in medical patients: a prospective study. J Hosp Med. 2011;6(2):61-67.

17. Steiner CA, Friedman B. Hospital utilization, costs, and mortality for adults with multiple chronic conditions, Nationwide Inpatient Sample, 2009. Prev Chronic Dis. 2013;10:E62.

18. Lochner KA, Cox CS. Prevalence of multiple chronic conditions among Medicare beneficiaries, United States, 2010. Prev Chronic Dis. 2013;10:E61.

19. Struijs JN, Baan CA, Schellevis FG, Westert GP, van den Bos GA. Comorbidity in patients with diabetes mellitus: impact on medical health care utilization. BMC Health Serv Res. 2006;6(1):84.

20. Berchick ER, Hood E, Barnett JC. Health insurance coverage in the United States: 2017: current population report. September 2018. Available at: https://www.census.gov/content/dam/Census/library/publications/2018/ demo/p60-264.pdf. Accessed March 21, 2019.

21. Munnell AH. The average retirement age-an update. March 2015. Available at: http://crr.bc.edu/wp-content/uploads/2015/03/IB_15-4.pdf. Accessed March 21, 2019.

22. Garthwaite C, Gross T, Notowidigdo MJ. Public health insurance, labor aupply, and employment lock. Quarterly J Econ. 2014;129(2):653-96.

23. Sommers BD, Gawande AA, Baicker K. Health insurance coverage and health-what the recent evidence tells us. N Engl J Med. 2017;377(6):586-93.

24. Sommers BD, Gunja MZ, Finegold K, Musco T. Changes in self-reported insurance overage, access to care, and health under the Affordable Care Act. JAMA. 2015;314(4):366-74C.

25. Chakraborty G, Ettenson R, Gaeth G. How consumers choose health insurance: analyzing employees' selection process in a multiplan environment identifies the trade-offs consumers make and the benefits that affect their decision making. J Health Care Mark. 1994;14(1):21-33.
26. U.S. Bureau of Labor Statistics. Employee tenure summary. September 20, 2018. Available at: https://www.bls.gov/news.release/tenure.nr0.htm. Accessed March 21, 2019.

27. Hendriks M, De Jong J, van den Brink-Muinen A, Groenewegen P. The intention to switch health insurer and actual switching behaviour: are there differences between groups of people? Health Expect. 2010;13(2):195-207.

28. Li Q, Trivedi AN, Galarraga O, Chernew ME, Weiner DE, Mor V. Medicare advantage ratings and voluntary disenrollment among patients with end-stage renal disease. Health Aff (Millwood). 2018;37(1):70-77.

29. Virnig BA, Morgan RO, DeVito CA, Persily NA. Medicare HMOs: who joins and who leaves? Am J Manag Care. 1998;4(4):511-18.

30. Temkin-Greener H, Bajorska A, Mukamel DB. Disenrollment from an acute/long-term managed care program (PACE). Med Care. 2006;44(1):31-38.

31. Pullmann MD, Heflinger CA, Mayberry LS. Patterns of Medicaid disenrollment for youth with mental health problems. Med Care Res Rev. 2010;67(6):657-75.

32. Chien LN, Adams EK. The effect of the Breast and Cervical Cancer Prevention and Treatment Act on Medicaid disenrollment. Womens Health Issues. 2010;20(4):266-71.

33. Elkin EB, Ishill N, Riley GF, et al. Disenrollment from Medicare managed care among beneficiaries with and without a cancer diagnosis. J Natl Cancer Inst. 2008;100(14):1013-21.

34. Field TS, Cernieux J, Buist D, et al. Retention of enrollees following a cancer diagnosis within health maintenance organizations in the Cancer Research Network. J Natl Cancer Inst. 2004;96(2):148-52.

35. Tarazi WW, Bradley CJ, Bear HD, Harless DW, Sabik LM. Impact of Medicaid disenrollment in Tennessee on breast cancer stage at diagnosis and treatment. Cancer. 2017;123(17):3312-19.

36. Huysse-Gaytandjieva A, Groot W, Pavlova M. A New perspective on job lock. Soc Indic Res. 2013;112(3):587-610.

37. Stroupe KT, Kinney ED, Kniesner JJ. Chronic illness and health insurance-related job lock. J Policy Anal Manage. 2001;20(3):525-44.

38. Kirchhoff AC, Nipp R, Warner EL, et al. "Job lock" among long-term survivors of childhood cancer: a report from the childhood cancer survivor study. JAMA Oncol. 2017;4(5):707-11. 
Health Plan Enrollment and Disenrollment of Individuals With and Without Established

Chronic Disease in a U.S. Commercially Insured and Medicare Advantage Population

\section{APPENDIX A Patient Attrition}

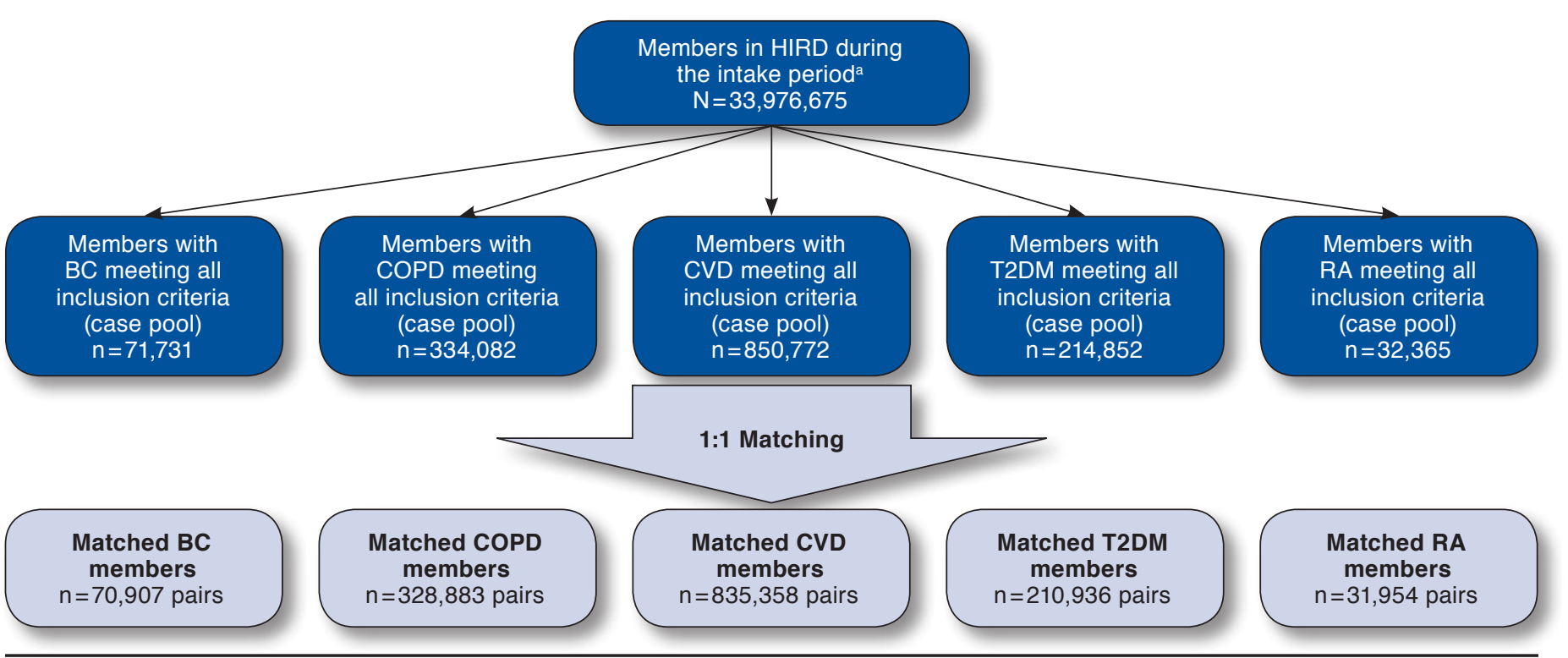

antake period: January 1, 2007-December 31, 2014.

$B C=$ breast cancer $; C O P D=$ chronic obstructive pulmonary disease $; C V D=$ cardiovascular disease; HIRD = HealthCore Integrated Research Database; $R A=$ rheumatoid arthritis; T2DM = type 2 diabetes mellitus. 
APPENDIX B Demographic Characteristics of BC, COPD, CVD, T2DM, and RA Case and Matched Control Cohorts

\begin{tabular}{|c|c|c|c|c|c|c|c|c|c|c|}
\hline & \multicolumn{2}{|c|}{$\mathrm{BC}$} & \multicolumn{2}{|c|}{ COPD } & \multicolumn{2}{|c|}{ CVD } & \multicolumn{2}{|c|}{ T2DM } & \multicolumn{2}{|c|}{ RA } \\
\hline Number of pairs, $n$ & \multicolumn{2}{|c|}{70,907} & \multicolumn{2}{|c|}{328,883} & \multicolumn{2}{|c|}{835,358} & \multicolumn{2}{|c|}{210,936} & \multicolumn{2}{|c|}{31,954} \\
\hline \multicolumn{11}{|l|}{ Age, mean (SD), years } \\
\hline & 60.5 & (13.3) & 66.7 & (13.2) & 62.7 & (14.3) & 53.6 & $(12.2)$ & 55.5 & $(14.5)$ \\
\hline \multicolumn{11}{|l|}{ Gender, n (\%) } \\
\hline Male & 235 & $(0.30)$ & 156,731 & $(47.70)$ & 413,283 & $(49.50)$ & 115,538 & $(54.80)$ & 8,954 & $(28.00)$ \\
\hline Female & 70,672 & $(99.70)$ & 172,152 & $(52.30)$ & 422,075 & $(50.50)$ & 95,398 & $(45.20)$ & 23,000 & $(72.00)$ \\
\hline \multicolumn{11}{|l|}{ Plan type, n (\%) } \\
\hline $\mathrm{HMO}$ & 15,252 & $(21.50)$ & 74,643 & $(22.70)$ & 185,880 & $(22.30)$ & 52,647 & $(25.00)$ & 7,291 & $(22.80)$ \\
\hline $\mathrm{PPO}$ & 50,821 & $(71.70)$ & 243,152 & $(73.90)$ & 610,957 & $(73.10)$ & 144,642 & $(68.60)$ & 22,642 & $(70.90)$ \\
\hline CDHP & 4,834 & $(6.80)$ & 11,088 & $(3.40)$ & 38,520 & $(4.60)$ & 13,647 & $(6.50)$ & 2,021 & $(6.30)$ \\
\hline Unknown & 0 & $(0.00)$ & 0 & $(0.00)$ & 1 & $(0.00)$ & 0 & $(0.00)$ & 0 & $(0.00)$ \\
\hline \multicolumn{11}{|l|}{ ACA plan, n (\%) } \\
\hline ACA exchange plan & 301 & $(0.40)$ & 529 & $(0.20)$ & 2,196 & $(0.30)$ & 805 & $(0.40)$ & 104 & $(0.30)$ \\
\hline Non-ACA plan & 70,606 & $(99.60)$ & 328,354 & $(99.80)$ & 833,162 & $(99.70)$ & 210,131 & $(99.60)$ & 31,850 & $(99.70)$ \\
\hline \multicolumn{11}{|c|}{ Residence region, $\mathrm{n}(\%)$} \\
\hline Northeast & 13,868 & $(19.60)$ & 57,649 & $(17.50)$ & 165,334 & $(19.80)$ & 32,984 & $(15.60)$ & 6,218 & $(19.50)$ \\
\hline Midwest & 21,335 & $(30.10)$ & 125,324 & $(38.10)$ & 275,465 & $(33.00)$ & 65,055 & $(30.80)$ & 9,301 & $(29.10)$ \\
\hline South & 18,775 & $(26.50)$ & 91,933 & $(28.00)$ & 226,023 & $(27.10)$ & 73,493 & $(34.80)$ & 9,087 & $(28.40)$ \\
\hline West & 16,929 & $(23.90)$ & 53,976 & $(16.40)$ & 168,528 & $(20.20)$ & 39,402 & $(18.70)$ & 7,347 & $(23.00)$ \\
\hline Othera & 0 & $(0.00)$ & 1 & $(0.00)$ & 8 & $(0.00)$ & 2 & $(0.00)$ & 1 & $(0.00)$ \\
\hline \multicolumn{11}{|c|}{ Primary policyholder, n (\%) } \\
\hline Primary holder & 54,220 & $(76.50)$ & 282,907 & $(86.00)$ & 698,658 & $(83.60)$ & 165,224 & $(78.30)$ & 23,705 & $(74.20)$ \\
\hline Dependent & 16,687 & $(23.50)$ & 45,976 & $(14.00)$ & 136,700 & $(16.40)$ & 45,712 & $(21.70)$ & 8,249 & $(25.80)$ \\
\hline \multicolumn{11}{|c|}{ Length of pre-index continuous health plan enrollment, $\mathrm{n}(\%)$} \\
\hline 12-17 months & 12,941 & $(18.30)$ & 69,600 & $(21.20)$ & 178,194 & $(21.30)$ & 46,770 & $(22.20)$ & 6,212 & $(19.40)$ \\
\hline $18-23$ months & 9,751 & $(13.80)$ & 48,849 & $(14.90)$ & 129,891 & $(15.50)$ & 32,470 & $(15.40)$ & 4,713 & $(14.70)$ \\
\hline 24-35 months & 14,497 & $(20.40)$ & 71,713 & $(21.80)$ & 184,763 & $(22.10)$ & 45,889 & $(21.80)$ & 6,976 & $(21.80)$ \\
\hline 36-47 months & 10,181 & $(14.40)$ & 46,692 & $(14.20)$ & 119,407 & $(14.30)$ & 30,115 & $(14.30)$ & 4,710 & $(14.70)$ \\
\hline $48-59$ months & 7,613 & $(10.70)$ & 32,188 & $(9.80)$ & 80,363 & $(9.60)$ & 20,551 & $(9.70)$ & 3,284 & $(10.30)$ \\
\hline$\geq 60$ months & 15,924 & $(22.50)$ & 59,841 & $(18.20)$ & 142,740 & $(17.10)$ & 35,141 & $(16.70)$ & 6,059 & $(19.00)$ \\
\hline \multicolumn{11}{|l|}{ Index year, n (\%) } \\
\hline 2007 & 11,584 & $(16.30)$ & 63,105 & $(19.20)$ & 162,315 & $(19.40)$ & 36,861 & $(17.50)$ & 5,138 & $(16.10)$ \\
\hline 2008 & 10,020 & $(14.10)$ & 53,145 & $(16.20)$ & 136,015 & $(16.30)$ & 32,151 & $(15.20)$ & 5,058 & $(15.80)$ \\
\hline 2009 & 9,509 & $(13.40)$ & 45,935 & $(14.00)$ & 117,986 & $(14.10)$ & 30,055 & $(14.20)$ & 4,652 & $(14.60)$ \\
\hline 2010 & 8,513 & $(12.00)$ & 39,264 & $(11.90)$ & 101,251 & $(12.10)$ & 27,165 & $(12.90)$ & 4,079 & $(12.80)$ \\
\hline 2011 & 8,600 & (12.10) & 37,462 & (11.40) & 93,273 & $(11.20)$ & 24,697 & (11.70) & 3,935 & (12.30) \\
\hline 2012 & 8,214 & (11.60) & 34,885 & $(10.60)$ & 84,602 & (10.10) & 21,644 & $(10.30)$ & 3,470 & (10.90) \\
\hline 2013 & 7,493 & $(10.60)$ & 30,187 & $(9.20)$ & 74,879 & $(9.00)$ & 19,657 & $(9.30)$ & 3,036 & $(9.50)$ \\
\hline 2014 & 6,974 & $(9.80)$ & 24,900 & $(7.60)$ & 65,037 & $(7.80)$ & 18,706 & $(8.90)$ & 2,586 & $(8.10)$ \\
\hline
\end{tabular}

a ther included armed forces overseas military locations, Canadian provinces, and U.S. territories and protectorates.

$A C A=$ Affordable Care Act; $B C=$ breast cancer $; C D H P=$ consumer-driven health plan; $C O P D=$ chronic obstructive pulmonary disease; $C V D=$ cardiovascular disease; $H M O=$ health maintenance organization; $P P O=$ preferred provider organization; $R A=$ rheumatoid arthritis; SD=standard deviation; T2DM=type 2 diabetes mellitus. 